\title{
Metalloproteinases in the pathogenesis and progression of metabolic syndrome: potential targets for improved outcomes
}

This article was published in the following Dove Press journal:

Metalloproteinases In Medicine

2I October 2015

Number of times this article has been viewed

\section{Gabriela Berg \\ Veronica Miksztowicz}

Lipids and Atherosclerosis Laboratory, Department of Clinical Biochemistry, Faculty of Pharmacy and Biochemistry, INFIBIOC, University of Buenos Aires, Buenos Aires, Argentina; National Scientific and Technical Research Council (CONICET), Mendoza, Argentina
Correspondence: Gabriela Berg Lipids and Atherosclerosis Laboratory, Department of Clinical Biochemistry, INFIBIOC, University of Buenos Aires Junín 956, CABA, Buenos Aires, Argentina

Tel +54 II4 9648297

$\mathrm{Fax}+54$ II 9508692

Email gaberg@ffyb.uba.ar
Abstract: Matrix metalloproteinases (MMPs) constitute a family of more than 25 calciumdependent, zinc-containing endopeptidases, synthesized by multiple cell types. These enzymes play an important role during physiological tissue development and remodeling and angiogenesis, as well as in pathophysiological conditions such as obesity and atherosclerotic process. Moreover, circulating levels of MMPs have emerged as potential biomarkers of cardiovascular disease. MMPs are regulated by different factors such as insulin resistance and obesity. Different components of the metabolic syndrome have been identified as possible stimulus for the synthesis and activity of MMPs. On the other hand, pro-inflammatory and anti-inflammatory cytokines, such as leptin and adiponectin, respectively, are associated with the regulation of MMPs. Leptin induces expression of MMP-2 activators as well as expression of MMP-2, MMP-9, and tissue inhibitor of metalloproteinase (TIMP)-1 in different human cells. Adiponectin may play a protective role in plaque rupture through selectively increasing TIMP expression. The hepatic manifestation of metabolic syndrome is nonalcoholic fatty liver disease (NAFLD). MMPs may remodel the liver parenchyma during the process of liver fibrosis. MMP-2 and MT1-MMP have been considered to be fibrogenic enzymes. There are few studies analyzing the role of MMPs in NAFLD, and most of them include study on mRNA expression, but even the results on their expression pattern remains controversial. MMPs could be considered as possible therapeutic targets. Different studies demonstrated that metformin, thiazolidinediones, and antibiotics could have inhibitory effects on the expression of MMPs; however, a rational study of lifestyle modifications as well as further studies of pharmacological therapies that influence MMPs are necessary to generate the information that physicians will probably need to improve the treatment of patients. The aim of this revision is to update the data about MMPs in the pathogenesis and progression of metabolic syndrome and the possible effect of different drugs on the behavior of these enzymes.

Keywords: metalloproteinases, metabolic syndrome, insulin resistance, nonalcoholic fatty liver, metformin, thiazolidinediones

\section{Introduction}

Matrix metalloproteinases (MMPs) constitute a family of more than 25 calciumdependent, zinc-containing endopeptidases, synthesized by multiple cell types. ${ }^{1}$ These enzymes present proteolytic function involved in the degradation of the components of extracellular matrix (ECM) and basement membranes (BMs), such as collagens, proteoglycans, elastin, laminin, fibronectin, and other glycoproteins. ${ }^{2}$ They can be classified according to their substrate specificity as collagenases, stromelysins, matrilysins, gelatinases, and membrane-type metalloproteinases. ${ }^{3}$ 
Most MMPs are secreted as precursor zymogens that are activated through a proteolytic process. Under physiological conditions, the activity of MMPs is regulated at the transcription level through interaction with specific ECM components and by inhibition of endogenous tissue inhibitors of MMPs (TIMPs). ${ }^{4,5}$ Consequently, the balance between activated MMPs and TIMPs locally determines the net result of the activity of MMPs in tissues.

MMPs play an important role during physiological tissue remodeling in embryonic development ${ }^{6}$ and angiogenesis, ${ }^{7}$ as well as in pathophysiological conditions such as atherosclerotic plaque development, and vulnerability ${ }^{8}$ and obesity. ${ }^{9}$ MMPs are known to be expressed in physiological conditions at some baseline level, but are differentially expressed in response to certain hormones, growth factors, and cytokines. ${ }^{10}$

Different studies have shown that increased levels of gelatinases (MMP-2 and MMP-9) are expressed in fatty streaks and atherosclerotic plaques, being partially responsible for plaques vulnerability. ${ }^{11,12}$ Regarding obesity, several MMPs have been studied in reference to the development of adipose tissue. Recent studies show that gelatinases are secreted by adipose tissue and that their activity is modulated during adipose tissue expansion/regression. ${ }^{13-15}$

\section{Metabolic syndrome}

Metabolic syndrome (MS) is a cluster of risk factors for cardiovascular disease (CVD) and type 2 diabetes mellitus (T2DM). According to the most used definition, the revised Adult Treatment Panel-III, ${ }^{16}$ MS is diagnosed when at least three of five of the following alterations are present: visceral obesity (waist circumference $>102 \mathrm{~cm}$ in men or $>88 \mathrm{~cm}$ in women); raised arterial blood pressure $(>13085 \mathrm{mmHg})$; dysglycemia (fasting plasma glucose $>100 \mathrm{mg} / \mathrm{dL}$ ); raised triglyceride concentrations ( $\geq 150 \mathrm{mg} / \mathrm{dL}$ ); low high-density lipoprotein cholesterol (HDL-chol $<40 \mathrm{mg} / \mathrm{dL}$ in men or $<50 \mathrm{mg} / \mathrm{dL}$ in women). MS confers a fivefold increase in the risk of T2DM and twofold increase in the risk of developing CVD over the next 5 to 10 years. ${ }^{17}$ Moreover, patients with MS are at two- to fourfold increased risk of developing a stroke, three- to fourfold increased risk of developing a myocardial infarction, and twofold increased risk of dying from such an event compared with those without the syndrome, ${ }^{18}$ regardless of a previous history of cardiovascular events. ${ }^{19}$ The pathophysiology of MS is very complex and not yet clear. Moreover, MS is associated with other systemic complications, such as obesity and fatty liver disease that affect different organs and organ systems. The expansion of adipose tissue is related to systemic inflammation through the secretion of adipokines in the systemic circulation resulting in a chronic inflammatory state, characteristic of MS.

\section{MMPs and MS}

Alterations of the arterial vasculature, especially of the endothelium, and basal membrane are characteristics of MS; other features associated with MS include polymorphonuclear activation, increased oxidative stress, and changes in the expression of MMPs. ${ }^{20}$ Different components of MS have been identified as possible stimuli for the synthesis and activity of MMPs. ${ }^{10}$ Hoseini et al found strong associations of MMP-8 with components of MS, suggesting that this MMP would be a potential cardiometabolic risk marker for MS, possibly through dependent and independent mechanisms of chronic low-grade inflammation. ${ }^{21}$ In our laboratory, we found increased MMP-2 plasma activity in women with $\mathrm{MS},{ }^{22}$ which correlates with vascular cell adhesion molecules involved in the plaque development. ${ }^{23}$ In accordance with the results obtained in other studies, Hopps et al showed an increase in plasma concentrations of MMP-2 and MMP-9 and their inhibitors (TIMP-1 and TIMP-2) in diabetic and nondiabetic subjects with MS. ${ }^{24}$ However, other authors found an increase in pro-MMP-9 and TIMP-1 levels, which are associated with raised concentrations of inflammatory markers and adhesion molecules, without differences in MMP-2 and TIMP-2 values in comparison with healthy controls. ${ }^{25}$ The different stages of the CVD could be a main factor in conditioning the circulating levels of MMPs. In a study by Miksztowicz et al, the patients included in the study were women with MS but without clinical evidence of unstable plaques. ${ }^{22}$ The increased MMP-2 activity could be associated with the first steps of the atherogenic process mainly related to the vascular smooth muscle cells (VSMCs) migration and intimal thickening. ${ }^{26}$ The lack of MMP-9 detection could be attributed to the fact that this MMP is reported to be associated mainly with the plaque rupture in advanced lesions. ${ }^{11}$ In turn, Opstad et al have shown that the MMP-9 $21562 \mathrm{C} / \mathrm{T}$ polymorphism modified the risk of new clinical events in patients with MS, partly mediated through altered MMP-9 regulation. Moreover, in patients with MS, the MMP-9 T-allele was associated with significantly increased risk of cardiovascular events and higher MMP-9 circulating levels, compared with non-MS patients. ${ }^{27}$

It is important to take into account that adipose tissue expansion could contribute to the circulating levels of MMPs, questioning the role of these enzymes as CVD biomarkers. In human adipose tissue from men with MS, a lack of 
association between adipose tissue mRNA and plasma levels of MMP-9 has been reported, suggesting that this tissue is not a major contributor to circulating MMP-9. ${ }^{28}$

In obesity, the expansion of adipose tissue is associated with modifications involving adipogenesis, angiogenesis, and ECM remodeling. MMPs are involved in the control of proteolysis and adipogenesis during obesity-mediated fat mass development (Figure 1), ${ }^{29}$ either through the degradation of ECM and BM components or by the activation of latent growth factors. ${ }^{14}$ Maquoi et al reported an upregulation of mRNA levels of some MMPs and TIMPs (MMP-3, MMP-11, MMP-12, MMP-13, and MMP-14 and TIMP-1) and downregulation of others (MMP-7, MMP-9, MMP-16, and MMP-24 and TIMP-4) in adipose tissue from lean and obese mice. ${ }^{30}$ These modulations differed according to the origin of the adipose tissue (gonadal vs subcutaneous), supporting the concept that different localization of fat deposits presents a different metabolic behavior. ${ }^{31}$

Through in vitro studies, Lijnen et al proposed that MMP-2 has a functional role in early and later stages of adipocyte differentiation, ${ }^{32}$ suggesting a possible function of MMP-2 in adipogenesis in vivo. On the contrary, in an in vivo animal model of insulin resistance induced by sucrose-rich diet, MMP-2 and MMP-9 activity was decreased in the visceral adipose tissue; however, this was not associated with changes in the activity of MMPs in plasma. ${ }^{33}$ The lack of association between adipose tissue and plasma activity of the gelatinases suggests that this tissue is not a major contributor of the circulating enzymes. In accordance with these results, Van Hul et al found that MMP-9 does not seem to play a major role in the adipose tissue development in murine models of diet-induced obesity. ${ }^{15}$ On the other hand, knockout models of different MMPs, such as MMP-19-null, MMP-3-null, or MMP-11-null mice, are associated with increase in adipose tissue development when mice are fed a high-fat diet. ${ }^{34-36}$ In contrast, in membrane type 1 MMP knockout model, visceral adipose tissue development is aborted, leaving tissues populated by mini-adipocytes which render null mice lipodystrophic. ${ }^{37}$ These controversies could be associated with not only the model used but also the stage of adipose tissue development.

\section{Effect of insulin resistance on MMP}

Insulin resistance causes hyperinsulinemia; therefore, practically all obese individuals, whether diabetic or not, are chronically hyperinsulinemic. Prospective studies found that

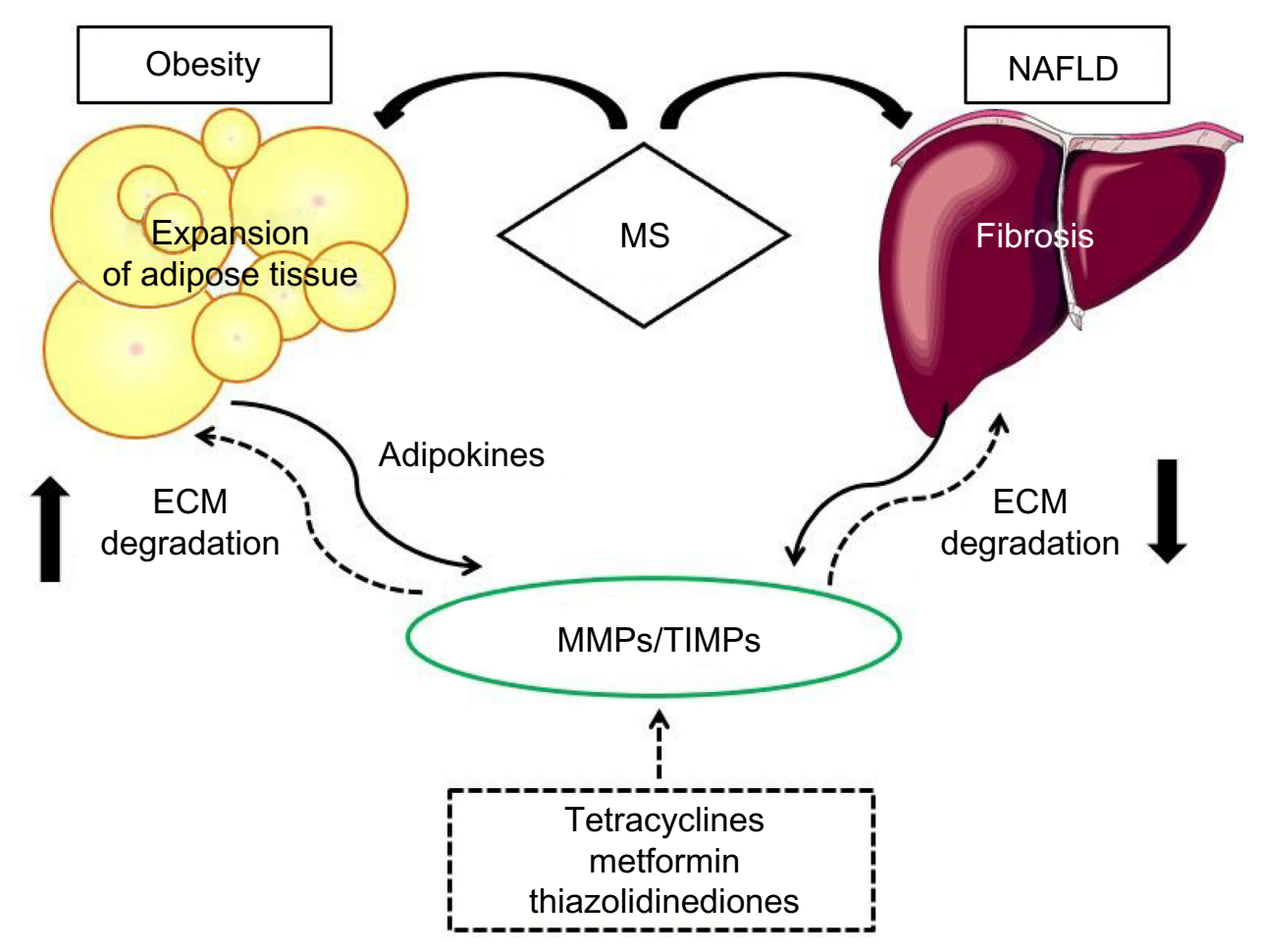

Figure I Relationship between MS, obesity and NAFLD, and MMPs/TIMPs balance.

Notes: MS is characterized by abdominal obesity and NAFLD. During the expansion of adipose tissue, there is an increase in the secretion of adipokines leading to an imbalance in MMP/TIMPs, which in turn promotes ECM degradation. In NAFLD, the increased expression of TIMPs and concomitantly the reduced activity of MMPs result in protein accumulation in the extracellular space and fibrosis. There is an evidence of the effect of tetracyclines, metformin, and thiazolidinediones on the activity of MMPs and TIMPs. Abbreviations: MS, metabolic syndrome; NAFLD, nonalcoholic fatty liver disease; MMPs, matrix metalloproteinases; TIMP, tissue inhibitor of metalloproteinase; ECM, extracellular matrix. 
elevated insulin levels were associated with an increased risk for ischemic heart disease, particularly in men, ${ }^{38,39}$ independent of the known CVD risk factors.

Early reports implicated insulin or insulin-like growth factor-1 signaling through the PI3K/Akt cascade as being responsible for the stimulation of MMP activity. ${ }^{40,41} \mathrm{In}$ vascular tissues, this pathway controls the metabolic actions of insulin, including glucose and amino acid uptake, glycogen synthesis, and nitric oxide (NO) production ${ }^{42}$ and is inhibited by free fatty acids in major insulin target tissues. ${ }^{43}$ Different studies have shown that insulin regulates MMPs in different ways according to the organ. Boden et al observed in rat aorta that free fatty acids and insulin promote the activation of MMP-2, MMP-9, and MT1-MMP through the stimulation of pro-inflammatory cytokines production. ${ }^{44}$ In contrast, in the liver, hyperinsulinemia promotes a decrease in MMP-2, MMP-9, and MT1-MMP levels. ${ }^{45}$

It has been shown that the expanded adipose tissue, characteristic of insulin-resistant state, presents areas of relative hypoxia. ${ }^{46}$ The development of hypoxia within white fat underlies the establishment of the inflammatory response in the tissue that leads to the diseases associated with obesity. Hypoxia-induced changes in adipokine production, having local effects, such as inflammation, or affecting other tissues, are associated with changes in the circulating level of the key adipocyte hormones leptin and adiponectin, as well as MMP-2. ${ }^{46}$

\section{Effects of cytokines on MMPs}

Leptin and adiponectin are the main cytokines synthesized in adipose tissue and exhibit different functions and opposing effects on inflammation and the atherosclerotic process. ${ }^{47}$

\section{Leptin}

Leptin was the first adipocytokine discovered in 1994, and its levels are increased in the plasma of obese individuals. Leptin shows central and peripheral effects. Central effects include the regulation of food intake and energy expenditure. ${ }^{48}$ Leptin also mediates a wide array of direct peripheral effects, including those specific to the cardiovascular system, given that its receptors are also present in many peripheral tissues. In vitro, leptin exhibits different proatherogenic effects, such as endothelial and VSMCs activation, migration, and proliferation. ${ }^{49,50}$ Leptin plays an important role in ECM remodeling by regulating the expression of MMPs and TIMPs. Previous studies in vitro have demonstrated stimulation of proteolytic activity of MMPs by leptin. ${ }^{51,52}$ It has been reported that exposure of myofibroblasts to leptin significantly increased the expression of MT1-MMP, resulting in an increase of MMP-2 activity, without changes in protein levels. ${ }^{53}$ Park et al reported that leptin induces elevation of MMP-2, MMP-9, and TIMP-1 expression in human umbilical vein endothelial cells and in human coronary artery smooth muscle cells ${ }^{49}$ through the generation of intracellular reactive oxidative species. In reference to adipose tissue, in vitro studies have demonstrated that MMP-2 secretion was significantly promoted by leptin treatment in 3T3-L1 preadipocytes. ${ }^{54}$ Because plasma leptin concentrations are associated with obesity and type 2 diabetes, ${ }^{55}$ leptin signaling may represent a therapeutic target for the prevention of obesity and CVD.

\section{Adiponectin}

Adiponectin is the most abundantly secreted adipokine in physiological situations, and its levels are inversely associated with obesity and inflammation. ${ }^{56}$ Adiponectin exerts well-known insulin-sensitizing effects and is also reported to possess beneficial effects on vascular function and may exhibit antiatherogenic and anti-inflammatory effects. The pathway by which adiponectin affects vascular function has been evaluated through in vitro experiments in endothelial cells from human aortas. Adiponectin increases NO production and/or ameliorates oxidized low-density lipoprotein (oxLDL)-induced suppression of endothelial NO synthase activity. ${ }^{57}$ Furthermore, adiponectin has been shown to affect atherosclerotic plaque formation and stability. ${ }^{56}$ Adiponectin suppresses lipid accumulation and class A scavenger receptor expression in macrophages, resulting in markedly decreased uptake of oxLDL and inhibition of foam cell formation. ${ }^{57}$ It also binds to platelet-derived growth factor-BB and subendothelial collagens and suppresses the proliferation and migration of human aortic smooth muscle cells. ${ }^{58}$

In vitro studies have shown that adiponectin exhibits different effects on the expression of different MMPs. Tong et al showed that adiponectin increased the secretion of MMP-3 in cultured human chondrocytes, ${ }^{59}$ whereas this cytokine reduced MMP-2 and MMP-9 protein levels of endometrial cancer cells.

Adiponectin may also play a protective role in plaque rupture through selectively increasing TIMP expression and secretion in human monocyte-derived macrophages. This effect is mediated via the ability of adiponectin to increase the expression and secretion of interleukin-10 (IL-10), a TIMP-inducing cytokine. ${ }^{60}$

Studies in humans show that in patients with combined hyperlipidemia, adiponectin decreased MMP-2, MMP-9, 
TIMP-1, and TIMP-2 plasma levels. ${ }^{61}$ In accordance with these results, in our laboratory we observed an inverse association between plasma adiponectin levels and circulating activity of MMP-2 in patients with MS, independently of obesity markers, suggesting that the inflammatory process associated with highest CVD risk would be involved in MMPs vascular production. ${ }^{62}$

Hypoadiponectinemia has been proposed to contribute to increased activity of MMP-2 in obese/hypertensive children and adolescents. ${ }^{63}$ Moreover, in patients with acute coronary syndrome, a negative relationship between adiponectin and MMP-9/TIMP-1 ratio has been described; this ratio is considered an independent predictor of atherosclerotic plaque stability and severity of coronary atherosclerosis. ${ }^{64}$ However, no correlations have been observed between adiponectin and plasma levels of MMP-1 in coronary patients. ${ }^{65}$ As has been mentioned previously, this could be a consequence of the behavior of MMPs according to cardiometabolic risk environments.

\section{MMPs and NAFLD}

NAFLD is defined as an excessive accumulation of triglycerides in the liver ( $>5 \%$ of hepatocytes histologically) in the absence of alcohol excess. ${ }^{66}$ NAFLD comprises a morphological spectrum of liver lesions ranging from simple triglyceride accumulation in hepatocytes (hepatic steatosis [HS]) to inflammatory and hepatocellular ballooning injury (nonalcoholic steatohepatitis [NASH]), eventually leading to fibrosis and cirrhosis. ${ }^{66}$

NAFLD is the leading cause of liver diseases in Western countries. ${ }^{67}$ The prevalence is increasing because of the rising occurrence of obesity and T2DM; ${ }^{66}$ in fact, NAFLD is considered as the hepatic manifestation of MS. ${ }^{68}$ NAFLD is present in $10 \%-24 \%$ of the general population in various countries, while the prevalence of NAFLD in obesity is $30 \%$ to $100 \%$ and in T2DM is $10 \%$ to $75 \%{ }^{69}$

In healthy liver, homeostasis of ECM is sustained by a precisely regulated permanent turnover directed by MMPs and TIMPs. Hepatic stellate cells (HSCs), localized in the perisinusoidal space, are the most important producers of ECM..$^{70}$ Upon chronic damage to liver tissue, HSCs become activated and differentiate into a fibroblast-like phenotype, increasing deposition of ECM. Therefore, expression of MMPs may vary during the progression of fibrotic liver disease (Figure 1). ${ }^{66}$

The activated HSCs produce very important cytokines, growth factors and inflammatory mediators, adhesion molecules (I-CAM-1, V-CAM-1, N-CAM), MMPs (MMP-1, -2,
$-3,-14$ ), and protease inhibitors (TIMP-1, -2 , plasminogen activator inhibitor-1), among other proteins. ${ }^{70}$ It has been reported that activated HSCs present increased expression of TIMP-1 and concomitantly reduced activity of MMPs with the subsequent protein accumulation, especially collagen type I, III, and IV, in the extracellular space. ${ }^{71}$ In murine models of cirrhosis and NASH, it has been reported the increase of expression and activity of MMP-2 and -9, respectively. ${ }^{72,73}$ HSCs secrete MMP-2 and MT1-MMP; the level of MMP-2 expression increases during the process of experimental hepatic fibrosis as well as during the process of hepatic fibrosis in chronic hepatitis, and it decreases during the process of cirrhosis. ${ }^{74}$ These MMPs may remodel the liver parenchyma during the process of liver fibrosis. Both MMP-2 and MT1-MMP have been considered to be fibrogenic enzymes because MMP-2 expression is stimulated by TGF- $\beta$ while MMP-1 expression is downregulated by TGF- $\beta .{ }^{75}$ Meanwhile, in human liver biopsies, no difference in MMP-9 activity was observed in steatosis situations. ${ }^{73}$ Anyway, there are few studies analyzing the role of MMPs in NAFLD, and most of them study mRNA expression, but even the results on their expression pattern remains controversial.

\section{MMPs as possible therapeutic targets Metformin and thiazolidinediones}

Metformin is a commonly prescribed oral anti-hyperglycemic drug used in the management of T2DM. Recent evidence indicates that metformin shows significant effects on tumorigenesis and cancer cell growth, and also exhibits antioxidant effects. It has been reported that patients with T2DM who take metformin have a lower risk of cancer than those who do not take metformin. ${ }^{76,77}$

Given the effect of metformin on tumorigenesis, special attention has been given to its effects on MMPs. Inhibitory action of metformin, a pharmacological activator of AMPK, on the MMP expression has been described in human fibrosarcoma cells. Hwang et al showed that metformin inhibits MMP-9 expression in human fibrosarcoma HT-1080 cells. ${ }^{78}$ Besides, Esfahanian et al have studied the effect of metformin on human umbilical vein endothelial cells, an established model for angiogenesis study. ${ }^{79}$ They observed that metformin significantly decreased MMP-2 and MMP-9 mRNA levels in a concentration-dependent manner. ${ }^{79}$ Furthermore, it has been demonstrated that metformin reduces MMP-2 expression in human aortic smooth muscle cells, which has been previously induced by leptin. ${ }^{49,51}$ Studies in animals showed that the combined effect of metformin and 
gemfibrozil reduces MMP-9 and increases adiponectin and IL-10 levels in streptozotocin-induced diabetes. ${ }^{80}$

Studies in humans have shown controversial results. In two different studies, it has been reported that metformin had no significant effect on serum MMP-9 levels after 3 and 6 months of treatment. ${ }^{81,82}$ However, Kiyici et al showed significant reductions in MMP-9 levels after 52 weeks of treatment, proposing that the beneficial effect of metformin on MMP-9 may result either from direct effects on endothelial cells or indirect actions such as improving glycemic control, promoting weight loss, and lowering the plasma levels of toxic compounds, such as glucose and free fatty acids, transported to the endothelium. ${ }^{83}$ All these studies included a reduced number of patients with T2MD; more recently, in a double blind, randomized, multicenter study with a similar number of patients, Hanefeld et al proposed that addition of pioglitazone but not of metformin reduces MMP-9 levels. ${ }^{84}$

Blaschke et al revised the effect of different thiazolidinediones, agonists to the peroxisome proliferators-activated receptor $\gamma$, and reported inhibition of MMP-9 expression and activity by roziglitazone and troglizatone independent of their antidiabetic action. ${ }^{85}$

Pfützner et al showed that pioglitazone downregulated the activated state of peripheral monocytes/macrophages

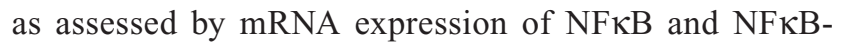
modulated cytokines and decreased plasma levels of MMP-9 independent of glycemic control. ${ }^{86}$ Different studies accord that pioglitazone is able to effectively suppress the expression of MMP-9 via a transcriptional mechanism; ${ }^{86,87}$ however, a recent meta-analysis of more than 700 patients revealed that neither roziglitazone nor pioglitazone had marked effects on MMP-9 concentrations. ${ }^{88}$

\section{Antibiotics}

The tetracyclines belong to a family of broad-spectrum antibiotics that inhibit protein synthesis by preventing the attachment of aminoacyl-tRNA to the ribosomal acceptor (A) site ${ }^{89}$ Besides, they present non-antimicrobial properties such as the ability to inhibit members of the MMP family (Figure 1). Although many synthetic compounds with the ability to inhibit MMP activity have entered clinical trials, ${ }^{90}$ none progressed to Phase III clinical trials. Doxycycline and minocycline are the most important semisynthetic tetracyclines used clinically as antibiotics. The mechanism by which tetracyclines inhibit MMPs has not been completely elucidated. A proposed mechanism is the direct inhibition of MMPs by the chelation of structural metals within the MMP. $^{89}$
After myocardial infarction, MMP activity is upregulated, suggesting an important role in mediating the acute injury and healing processes of the heart. However, early activation of MMP could also lead to early damage of the cardiac ECM. After ischemia reperfusion injury, MMP-2 activity increases in isolated, perfused rat hearts resulting in proteolysis of troponin I and myosin light chain..$^{91,92}$ The treatment with doxycycline attenuated the increase in MMP-2 activity upon reperfusion and improved the recovery of contractile function. ${ }^{93,94}$ Inhibition of MMP activity holds promise for preserving cardiac structure and function post-myocardial infarction, however, important being the timing of this inhibition. Significant anti-remodeling (ie, beneficial) effects can be observed in the infarcted heart when doxycycline is given for a limited period of time after injury. ${ }^{95}$

\section{Conclusion}

As we have reviewed, there is a bidirectional interaction between insulin resistance, a characteristic feature of MS that through different signaling pathways increases the production and activation of MMPs, and MMPs contributing to the development and expansion of adipose tissue with an increase in insulin-resistant state. Other signaling pathways, such as elevated leptin and decreased adiponectin production, also participate in the misbalance between MMPs and TIMPs. The synergistic interaction among these risk factors contributes to the development of heart disease. Given the proved role of MMPs on atherosclerotic plaque vulnerability, a tangled web of different risk factors appears to play a role in accelerating CVD. Pharmacological treatment has started to provide new and promising results for the treatment of MS. Further studies regarding lifestyle modifications as well as pharmacological therapies are necessary to improve the treatment of patients.

\section{Acknowledgment}

This work was supported by a grant from the National Agency of Promotion of Science and Technology (No PICT 2013-1150).

\section{Disclosure}

The authors report no conflicts of interest in this work.

\section{References}

1. Newby AC. Dual role of matrix metalloproteinases (matrixins) in intimal thickening and atherosclerotic plaque rupture. Physiol Rev. 2005;85: $1-31$.

2. Nagase H, Visse R, Murphy G. Structure and function of matrix metalloproteinases and TIMPs. Cardiovasc Res. 2006;69:562-573. 
3. Borkakoti N. Structural studies of matrix metalloproteinases. $J$ Mol Med. 2000;78:261-268.

4. Nagase H. Metalloproteases. Curr Protoc Protein Sci. 2011; Chapter 21(Unit 21.4):1-13.

5. Baker AH, Edwards DR, Murphy G. Metalloproteinase inhibitors: biological actions and therapeutic opportunities. J Cell Sci. 2002;115: 3719-3727.

6. Vu TH, Werb Z. Matrix metalloproteinases: effectors of development and normal physiology. Genes Dev. 2000;14:2123-2133.

7. Roy R, Zhang B, Moses MA. Making the cut: protease-mediated regulation of angiogenesis. Exp Cell Res. 2006;312:608-622.

8. Newby AC. Metalloproteinases and vulnerable atherosclerotic plaques. Trends Cardiovasc Med. 2007;17:253-258.

9. Lijnen HR. Murine models of obesity and hormonal therapy. Thromb Res. 2011;127:S17-S20

10. Berg G, Miksztowicz V, Schreier L. Metalloproteinases in metabolic syndrome. Clin Chim Acta. 2011;412:1731-1739.

11. de Nooijer R, Verkleij CJ, von der Thüsen JH, et al. Lesional overexpression of matrix metalloproteinase- 9 promotes intraplaque hemorrhage in advanced lesions but not at earlier stages of atherogenesis. Arterioscler Thromb Vasc Biol. 2006;26:340-346.

12. Choudhary S, Higgins CL, Chen IY, et al. Quantitation and localization of matrix metalloproteinases and their inhibitors in human carotid endarterectomy tissues. Arterioscler Thromb Vasc Biol. 2006;26:2351-2358.

13. Bouloumie A, Sengenes C, Portolan G, Galitzky J, Lafontan M. Adipocyte produces matrix metalloproteinases 2 and 9: involvement in adipose differentiation. Diabetes. 2001;50:2080-2086.

14. Lijnen HR, Maquoi E, Holvoet P, et al. Adipose tissue expression of gelatinases in mouse models of obesity. Thromb Haemost. 2001;85: $1111-1116$.

15. Van Hul M, Lijnen HR. Effect of weight loss on gelatinase levels in obese mice. Clin Exp Pharmacol Physiol. 2011;38:647-649.

16. Grundy SM, Cleeman JI, Merz CN, et al. Implications of recent clinical trials for the National Cholesterol Education Program Adult Treatment Panel III Guidelines. Circulation. 2004;110:227-239.

17. Alberti KG, Eckel RH, Grundy SM, et al. Harmonizing the metabolic syndrome: a joint interim statement of the international diabetes federation task force on epidemiology and prevention; National heart, lung, and blood institute; American heart association; World heart federation; International atherosclerosis society; and International Association for the Study of Obesity. Circulation. 2009;120:1640-1645.

18. Alberti KG, Zimmet P, Shaw J. The metabolic syndrome - a new worldwide definition. Lancet. 2005;366:1059-1062.

19. Olijhoek JK, van der Graaf Y, Banga JD, Algra A, Rabelink TJ, Visseren FL. The metabolic syndrome is associated with advanced vascular damage in patients with coronary heart disease, stroke, peripheral arterial disease or abdominal aortic aneurysm. Eur Heart $J$. 2004;25:342-348.

20. Hopps E, Caimi G. Matrix metaloproteases in metabolic syndrome. Eur J Intern Med. 2012;23:99-104.

21. Hoseini SM, Kalantari A, Afarideh M, et al. Evaluation of plasma MMP-8, MMP-9 and TIMP-1 identifies candidate cardiometabolic risk marker in metabolic syndrome: results from double-blinded nested case-control study. Metabolism. 2015;64:527-538.

22. Miksztowicz V, Muzzio ML, Royer M, et al. Increased plasma activity of metalloproteinase 2 in women with metabolic syndrome. Metabolism. 2008;57:1493-1496.

23. Muzzio ML, Miksztowicz V, Repetto EM, Brites F, Berg G, Schreier L. Increased MMP-2 in healthy postmenopausal women. Ann Clin Biochem. 2012;49:75-79.

24. Hopps E, Lo Presti R, Montana M, Noto D, Averna MR, Caimi G. Gelatinases and their tissue inhibitors in a group of subjects with metabolic syndrome. J Investig Med. 2013;61:978-983.

25. Gonçalves FM, Jacob-Ferreira AL, Gomes VA, et al. Increased circulating levels of matrix metalloproteinase (MMP)-8, MMP-9, and pro-inflammatory markers in patients with metabolic syndrome. Clin Chim Acta. 2009;403:173-177.
26. Johnson JL. Matrix metalloproteinases: influence on smooth muscle cells and atherosclerotic plaque stability. Expert Rev Cardiovasc Ther. 2007;5:265-282.

27. Opstad TB, Arnesen H, Pettersen A, Seljeflot I. The MMP-9 -1562 C/T polymorphism in the presence of metabolic syndrome increases the risk of clinical events in patients with coronary artery disease. PLoS One. 2014;9:e106816.

28. Gummesson A, Hagg D, Olson FJ, Hulthe J, Carlsson LM, Fagerberg B. Adipose tissue is not an important source for matrix metalloproteinase-9 in the circulation. Scand J Clin Lab Invest. 2009;69:636-642.

29. Boden G. Obesity and free fatty acids. Endocrinol Metab Clin North Am. 2008;37:635-646.

30. Maquoi E, Munaut C, Colige A, Collen D, Lijnen HR. Modulation of adipose tissue expression of murine matrix metalloproteinases and their tissue inhibitors with obesity. Diabetes. 2002;51:1093-1101.

31. Christiaens V, Lijnen HR. Role of the fibrinolytic and matrix metalloproteinase systems in development of adipose tissue. Arch Physiol Biochem. 2006;112:254-259.

32. Lijnen HR, Maquoi E, Hansen LB, Van Hoef B, Frederix L, Collen D. Matrix metalloproteinase inhibition impairs adipose tissue development in mice. Arterioscler Thromb Vasc Biol. 2002;22:374-379.

33. Miksztowicz V, Morales C, Zago V, Friedman S, Schreier L, Berg G. Effect of insulin-resistance on circulating and adipose tissue MMP-2 and MMP-9 activity in rats fed a sucrose-rich diet. Nutr Metab Cardiovasc Dis. 2014;24:294-300.

34. Pendás AM, Folgueras AR, Llano E, et al. Diet-induced obesity and reduced skin cancer susceptibility in matrix metalloproteinase 19-deficient mice. Mol Cell Biol. 2004;24:5304-5313.

35. Maquoi E, Demeulemeester D, Voros G, Collen D, Lijnen HR. Enhanced nutritionally induced adipose tissue development in mice with stromelysin-1 gene inactivation. Thromb Haemost. 2003;89: 696-704.

36. Lijnen HR, Van HB, Frederix L, Rio MC, Collen D. Adipocyte hypertrophy in stromelysin-3 deficient mice with nutritionally induced obesity. Thromb Haemost. 2002;87:530-535.

37. Chun TH, Inoue M, Morisaki H, et al. Genetic link between obesity and MMP14-dependent adipogenic collagen turnover. Diabetes. 2010;59: 2484-2494.

38. Despres JP, Lamarchie B, Mauriège $\mathrm{P}$, et al. Hyperinsulinemia as an independent risk factor for ischemic heart disease. $N$ Engl $J$ Med. 1996;334:952-957.

39. Pyoralla K. Relationship of glucose tolerance and plasma insulin to the incidence of coronary heart disease: results from two population studies in Finland. Diabetes Care. 1979;2:131-141.

40. Lee MPS, Sweeney G. Insulin increases gelatinase activity in rat glomerular mesangial cells via ERK- and PI-3 kinase-dependent signalling. Diabetes Obes Metab. 2005;8:281-288.

41. Risinger GM, Hunt TS, Updike DL, Bullen EC, Howard EW. Matrix metalloproteinase-2 expression by vascular smooth muscle cells is mediated by both stimulatory and inhibitory signals in response to growth factors. J Biol Chem. 2006;281:25915-25925.

42. Saltiel AR, Kahn CR. Insulin signaling and the regulation of glucose and lipid metabolism. Nature. 2001;414:799-806.

43. Boden G. Role of fatty acids in the pathogenesis of insulin resistance and NIDDM. Diabetes. 1997;46:3-10.

44. Boden G, Song W, Pashko L, Kresge K. In vivo effects of insulin and free fatty acids on matrix metalloproteinases in rat aorta. Diabetes. 2008;57:476-483.

45. Boden G, Song W, Kresge K, Mozzoli M, Cheung P. Effects of hyperinsulinemia on hepatic metalloproteinases and their tissue inhibitors. Am J Physiol Endocrinol Metab. 2008;295:e692-e697.

46. Trayhurn P, Alomar SY. Oxygen deprivation and the cellular response to hypoxia in adipocytes - perspectives on white and brown adipose tissues in obesity. Front Endocrinol. 2015;6:19.

47. Ntaios G, Gatselis NK, Makaritsis K, Dalekos GN. Adipokines as mediators of endothelial function and atherosclerosis. Atherosclerosis. 2013;227:216-221. 
48. Sweeney G. Leptin signaling. Cell Signal. 2002;14:655-663.

49. Park HY, Kwon HM, Lim HJ, et al. Potential role of leptin in angiogenesis: leptin induces endothelial cell proliferation and expression of matrix metalloproteinases in vivo and in vitro. Exp Mol Med. 2001;33: 95-102.

50. Oda A, Taniguchi T, Yokoyama M. Leptin stimulates rat aortic smooth muscle cell proliferation and migration. Kobe J Med Sci. 2001;47:141-150

51. Li L, Mamputu JC, Wiernsperger N, Renier G. Signaling pathways involved in human vascular smooth muscle cell proliferation and matrix metalloproteinase-2 expression induced by leptin: inhibitory effect of metformin. Diabetes. 2005;54:2227-2234.

52. Madani S, De Girolamo S, Munoz DM, Li RK, Sweeney G. Direct effects of leptin on size and extracellular matrix components of human pediatric ventricular myocytes. Cardiovasc Res. 2005;69: 716-725.

53. Schram K, Wong MM, Palanivel R, No EK, Dixon IM, Sweeney G. Increased expression and cell surface localization of MT1-MMP plays a role in stimulation of MMP-2 activity by leptin in neonatal rat cardiac myofibroblasts. J Mol Cell Cardiol. 2008;44:874-881

54. Moon HS, Lee HG, Seo JH, et al. Leptin-induced matrix metalloproteinase- 2 secretion I suppressed by trans-10,cis-12 conjugated linoleic acid. Biochem Biophys Res Commun. 2007;356:955-956.

55. Reilly MP, Iqbal N, Schutta M, et al. Plasma leptin levels are associated with coronary atherosclerosis in type 2 diabetes. J Clin Endocrinol Metab. 2004;89:3872-3878.

56. Villarreal-Molina MT, Antuna-Puente B. Adiponectin: anti-inflammatory and cardioprotective effects. Biochimie. 2012;94:2143-2149.

57. Matsuzawa Y. Adiponectin: identification, physiology and clinical relevance in metabolic and vascular disease. Atheroscler Suppl. 2005;6:7-14.

58. Arita Y, Kihara S, Ouchi N, et al. Adipocyte-derived plasma protein adiponectin acts as a platelet-derived growth factor-BB-binding protein and regulates growth factor-induced common postreceptor signal in vascular smooth muscle cell. Circulation. 2002;105: 2893-2898.

59. Tong KM, Chen CP, Huang KC, et al. Adiponectin increases MMP-3 expression in human chondrocytes through AdipoR1 signaling pathway. J Cell Biochem. 2011;112:1431-1440.

60. Kumada M, Kihara S, Ouchi N, et al. Adiponectin specifically increased tissue inhibitor of metalloproteinase-1 through interleukin-10 expression in human macrophages. Circulation. 2004;109:2046-2049.

61. Derosa G, Maffioli P, D'Angelo A, et al. Evaluation of metalloproteinase 2 and 9 levels and their inhibitors in combined dyslipidemia. Clin Invest Med. 2009;32:E124-E132.

62. Miksztowicz V, Fernandez Machulsky N, Lucero D, Fassio E, Schreier L, Berg G. Adiponectin predicts MMP-2 activity independently of obesity. Eur J Clin Invest. 2014;44:951-957.

63. Belo VA, Lacchini R, Miranda JA, Lanna CM, Souza-Costa DC, Tanus-Santos JE. Increased activity of MMP-2 in hypertensive obese children is associated with hypoadiponectinemia. Obesity. 2015;23: $177-182$.

64. Cheng M, Hashmi S, Mao X, Zeng QT. Relationships of adiponectin and matrix metalloproteinase- 9 to tissue inhibitor of metalloproteinase-1 ratio with coronary plaque morphology in patients with acute coronary syndrome. Can J Cardiol. 2008;24:385-390.

65. Hwang J, Yang W, Chiang F, et al. Association of circulating matrix metalloproteinase-1, but not adiponectin, with advanced coronary artery disease. Atherosclerosis. 2009;204:293-297.

66. Farrell GC, Larter CZ. Nonalcoholic fatty liver disease: from steatosis to cirrhosis. Hepatology. 2006;43:s99-s112.

67. Angulo P. GI epidemiology: nonalcoholic fatty liver disease. Aliment Pharmacol Ther. 2007;25:883-889.

68. Musso G, Gambino R, Bo S, et al. Should nonalcoholic fatty liver disease be included in the definition of metabolic syndrome? A cross-sectional comparison with adult treatment panel III criteria in non-obese nondiabetic subjects. Diabetes Care. 2008;31(3):562-568.
69. Ahmed MH, Barakat S, Almobarak AO. Nonalcoholic fatty liver disease and cardiovascular disease: has the time come for cardiologists to be hepatologists? J Obes. 2012;2012:483135.

70. Friedman SL. Seminars in medicine of the Beth Israel Hospital, Boston. The cellular basis of hepatic fibrosis. Mechanisms and treatment strategies. N Eng J Med. 1993;328:1828-1835.

71. Hemmann S, Graf J, Roderfeld M, Roeb E. Expression of MMPs and TIMPs in liver fibrosis a systematic review with special emphasis on anti-fibrotic strategies. J Hepatol. 2007;46:955-975.

72. Knittel T, Mehde M, Grundmann A, Saile B, Scharf J, Ramadori G. Expression of matrix metalloproteinases and their inhibitors during hepatic tissue repair in the rat. Histochem Cell Biol. 2000;113:443-453.

73. Wanninger J, Walter R, Bauer S, et al. MMP-9 activity is increased by adiponectin in primary human hepatocytes but even negatively correlates with serum adiponectin in a rodent model of non-alcoholic steatohepatitis. Exp Mol Pathol. 2011;91:603-607.

74. Takahara T, Furui K, Funaki J, et al. Increased expression of matrix metalloproteinase-II in experimental liver fibrosis in rats. Hepatology. 1995;21:787-795.

75. Okazaki I, Nabeshima K. Introduction: MMPs, ADAMs/ADAMTSs research products to achieve big dream. Anticancer Agents Med Chem. 2012;12:688-706

76. Evans JM1, Donnelly LA, Emslie-Smith AM, Alessi DR, Morris AD. Metformin and reduced risk of cancer in diabetic patients. $\mathrm{Br}$ Med $\mathrm{J}$. 2005;330:1304-1305.

77. Bowker SL1, Majumdar SR, Veugelers P, Johnson JA. Increased cancerrelated mortality for patients with type 2 diabetes who use sulfonylureas or insulin. Diabetes Care. 2006;29:254-258.

78. Hwang YP, Jeong HG. Metformin blocks migration and invasion of tumour cells by inhibition of matrix metalloproteinase- 9 activation through a calcium and protein kinase Calpha-dependent pathway: phorbol-12-myristate-13-acetate-induced/extracellular signal-regulated kinase/activator protein-1. Br J Pharmacol. 2010;160:1195-1211.

79. Esfahanian N, Shakiba Y, Nikbin B, et al. Effect of metformin on the proliferation, migration, and MMP-2 and -9 expression of human umbilical vein endothelial cells. Mol Med Rep. 2012;5:1068-1074.

80. Sharma AK, Raikwar SK, Kurmi MK, Srinivasan BP. Gemfibrozil and its combination with metformin on pleiotropic effect on IL-10 and adiponectin and anti-atherogenic treatment in insulin resistant type 2 diabetes mellitus rats. Inflammopharmacology. 2013;21:137-145.

81. Goldstein BJ, Weissman PN, Wooddell MJ, Waterhouse BR, Cobitz AR. Reductions in biomarkers of cardiovascular risk in type 2 diabetes with rosiglitazone added to metformin compared with dose escalation of metformin: an EMPIRE trial sub-study. Curr Med Res Opin. 2006; 22(9):1715-1723.

82. Huang Z, Lei MX, Liu L, Tang QB. Effects of rosiglitazone on the IMTc and serum MMP-9 levels in newly diagnosed type 2 diabetic patients. Zhong Nan Da Xиe Xие Bao Yi Xиe Ban. 2006;31:367-372.

83. Kiyici S1, Ersoy C, Kaderli A, et al. Effect of rosiglitazone, metformin and medical nutrition treatment on arterial stiffness, serum MMP-9 and MCP-1 levels in drug naive type 2 diabetic patients. Diabetes Res Clin Pract. 2009;86:44-50.

84. Hanefeld M, Pfützner A, Forst T, Kleine I, Fuchs W. Double-blind, randomized, multicentre, and active comparator controlled investigation of the effect of pioglitazone, metformin, and the combination of both on cardiovascular risk in patients with type 2 diabetes receiving stable basal insulin therapy: the PIOCOMB study. Cardiovasc Diabetol. 2011;10:65.

85. Blaschke F, Spanheimer R, Khan M, Law RE. Vascular effects of TZDs: new implications. Vascul Pharmacol. 2006;45:3-18.

86. Pfützner A, Weise A, Pfützner-Riehn E, et al. Downregulation of the proinflammatory state of circulating mononuclear cells by short-term treatment with pioglitazone in patients with type 2 diabetes mellitus and coronary artery disease. PPAR Res. 2011;2011:647017.

87. Zhang J, Huang X, Wang L. Pioglitazone inhibits the expression of matrix metalloproteinase- 9 , a protein involved in diabetes-associated wound healing. Mol Med Rep. 2014;10:1084-1088. 
88. Chen R, Yan J, Liu P, Wang Z. Effects of thiazolidinedione therapy on inflammatory markers of type 2 diabetes: a meta-analysis of randomized controlled trials. PLoS One. 2015;10:e0123703.

89. Griffin MO, Fricovsky E, Ceballos G, Villarreal F. Tetracyclines: a pleitropic family of compounds with promising therapeutic properties. Review of the literature. Am J Physiol Cell Physiol. 2010;299:C539-C548.

90. Peterson JT. Matrix metalloproteinase inhibitor development and the remodeling of drug discovery. Heart Fail Rev. 2004;9:63-79.

91. Sawicki G, Leon H, Sawicka J, et al. Degradation of myosin light chain in isolated rat hearts subjected to ischemia- reperfusion injury: a new intracellular target for matrix metalloproteinase-2. Circulation. 2005;112:544-552.
92. Wang W, Schulze CJ, Suarez-Pinzon WL, Dyck JRB, Sawicki G, Schulz R. Intracellular action of matrix metalloproteinase-2 accounts for acute myocardial ischemia and reperfusion injury. Circulation. 2002;106:1543-1549.

93. Donato M, D'Annunzio V, Buchholz B, et al. Role of matrix metalloproteinase-2 in the cardioprotective effect of ischaemic postconditioning. Exp Physiol. 2010;95:274-281.

94. Cheung PY, Sawicki G, Wozniak M, Wang W, Radomski MW, Schulz R Matrix metalloproteinase-2 contributes to ischemia-reperfusion injury in the heart. Circulation. 2000;101:1833-1839.

95. Garcia RA, Go KV, Villarreal FJ. Effects of timed administration of doxycycline or methylprednisolone on post-myocardial infarction inflammation and left ventricular remodeling in the rat heart. Mol Cell Biochem. 2007;300:159-169.

\section{Publish your work in this journal}

Metalloproteinases In Medicine is an international, peer reviewed, open access journal that aims to provide a platform for the discussion and dissemination of knowledge about the role that metalloproteinases - such as matrix metalloproteinases (MMP), ADAMs, ADAMTSs, and astacins, as well as their inhibitors - play in diseases.

\section{Dovepress}

The manuscript management system is completely online and includes a very quick and fair peer review system, which is all easy to use. Visit http://www.dovepress.com/testimonials.php to read real quotes from published authors.

Submit your manuscript here: http://www.dovepress.com/metalloproteinases-in-medicine-journal 